\title{
High resolution stellar kinematics for NGC4650A: solving the enigma of the flattening of its dark halo
}

\author{
Enrichetta lodice ${ }^{* \dagger}$ \\ INAF - Osserv. Astron. di Capodimonte, Italy \\ E-mail: iodice@na.astro.it

\section{Arnaboldi} \\ INAF - Osservatorio Astronomico di Torino \\ E-mail: arnaboldi@to.astro.it
}

\begin{abstract}
We have obtained high resolution rotation curves and velocity dispersion profiles along the major and minor axis of the central galaxy in the polar ring galaxy NGC4650A. These data are now crucial to derive constraints on the 3-D shape of the dark halo in this peculiar object, given the new results obtained by analysing the NIR and HST imaging data available for this system.
\end{abstract}

Baryons in Dark Matter Halos

5-9 October 2004

Novigrad, Croatia

\footnotetext{
* Speaker.

${ }^{\dagger}$ We are grateful to R. Saglia for useful discussions and for the help in the use of FCQ program.
} 


\section{Scientific background}

The Polar Ring Galaxy (PRG) NGC4650A (Fig.1) is considered the prototype of the class of wide PRGs: the central host galaxy (HG) is surrounded by a bright and well defined ring, made up by gas, stars and dust, which orbit nearly perpendicular to the plane of the central galaxy. The existence of two orthogonal components of the angular momentum in PRGs make these peculiar galaxies a unique laboratory to determine the 3D shape of the dark matter halos around galaxies. To this aim, NGC4650A is one of the best-investigated PRGs (Sackett et al. 1994; Combes \& Arnaboldi 1996; Iodice et al. 2003). The biggest uncertainties in the mass model proposed until 1996 for the dark halo shape resulted from the low resolution of the HI line 21-cm observations available at that time and the difficulty in measuring velocity dispersion profiles along the major axis of the HG. The new high resolution 21-cm observations (M. Arnaboldi et al. 1997, which give velocity field along the polar direction to about $40 \mathrm{kpc}$ from the galaxy center) and the new surface photometry available for NGC4650A, based on new NIR (in J, H and Kn bands) and optical HST data (Iodice et al. 2002; Gallagher et al. 2002), suggested that the polar structure appears to be a disk, which is characterized by a very young age, and the HG has a most peculiar structure respect to the standard early-type systems. In the light of these new data and results, a revised dynamical model for the HI, optical structure and kinematics of NGC4650A should be done. To this aim, deep long slit spectroscopy along the photometric axes of central stellar component of NGC4650A have been obtained: data were obtained with FORS2 at ESO UT4-VLT (8.2m) telescope, during several observing runs (January - April 2003). The adopted grism covers the wavelength range 773-948 $\mathrm{nm}$, centered at $859 \mathrm{~nm}$ and a dispersion of $0.086 \mathrm{~nm} /$ pixel. In this wavelength range are located the absorptions features of the Calcium triplet $(848.9-866.2 \mathrm{~nm})$. For all observations the slit width is 1 ". 6 and the slit length is 6'.8.

The Line-of-Sight Velocity Distribution (LOSVD) was described by a Gaussian plus third- and fourth-order Gauss-Hermite functions; the LOSVD and kinematic parameters were derived from the continuum-removed spectra by using the Fourier Correlation Quotient (FCQ) method (Bender, Saglia \& Gerhard 1994).

\section{Results}

In Fig. 1 we plot the rotation curve and velocity dispersion relative to the major and minor axis of the HG in NGC4650A.

Along the major axis the rotation curve is extended to about 2 times the disk scalelenght, which is about 5.9 arcsec. The velocity is slowly increasing and reaches the maximum value of about 90 $\mathrm{km} / \mathrm{s}$ in the NE at about 10 arcsec from the center, this value is consistent with the maximum value derived by Sackett et al. (1994) and with that derived by Swaters \& Rubin (2003). The SW rotation velocities in the range 1 to about 3 arcsec show a depletion which is absent in the NE velocities; this is reasonably due to the contamination by the velocities of stars in the PR arm which passing in front of HG in this region (see Fig. 2 in Iodice et al. 2002). The velocity dispersion has a central value of about $68.8 \mathrm{~km} / \mathrm{s}$ and it is almost constant, $\sigma \sim 73 \mathrm{~km} / \mathrm{s}$, till about 6 arcsec. For larger radii it increases and reaches a value of about $140 \mathrm{~km} / \mathrm{s}$ at $11 \mathrm{arcsec}$.

Along the minor axis the slit length is much more extended than the HG (that reaches about 4-5

$081 / 2$ 

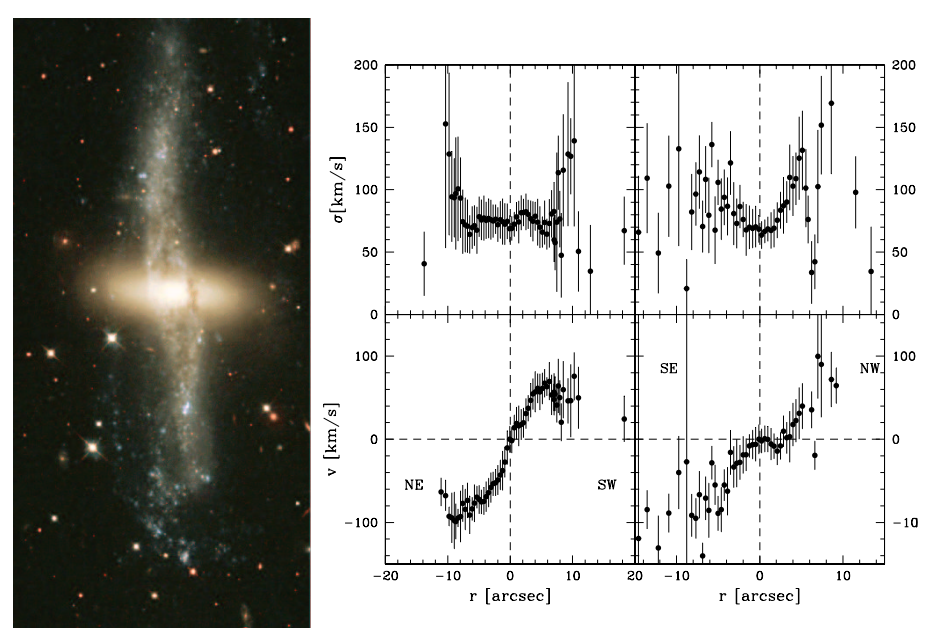

Figure 1: Left - Polar Ring Galaxy NGC4650A: color composite image by the HST heritage project. North is almost up and East is to the left. Right - Rotation curve and velocity dispersion along the major (left panel) and minor axis (right panel) of the HG in NGC4650A.

arcsec, see Iodice et al. 2002), so the derived spectra includes also light coming from some regions of the polar ring and this explains the increasing rotation till about $110 \mathrm{~km} / \mathrm{s}$ at $7 \mathrm{arcsec}$. The velocity dispersion is almost constant (at about $69 \mathrm{~km} / \mathrm{s}$ ) till about 2 arcsec from the galaxy center, then it rises and reaches a value of about $140 \mathrm{~km} / \mathrm{s}$ at about $5 \mathrm{arcsec}$.

The absence of a central peak and the constancy of the velocity dispersion suggest that the inner regions of the $\mathrm{HG}$ in NGC4650A is dominated by the kinematics of a cold disk. By taking into account the results obtained by photometry (Iodice et al. 2002), HI data (Arnaboldi et al. 1997) we think that, along the major axis, the constancy of dispersion from the center to about 6 arcsec can be explain just as the presence of this disk. For radii larger than 6 arcsec, we may observe the "true" values of the dispersion for the central galaxy. Along the minor axis, taking into account the apparent axial ratio, which is about 0.5 , we expect that the polar disk will influence the HG kinematics till about 3 arcsec: this is just what we observe. These results can be considered as the kinematic evidence of the polar disk in NGC4650A and will be take into account to derive an accurate mass model for NGC4650A.

\section{References}

[1] Arnaboldi et al. 1997 AJ 113, 585

[2] Bender, Saglia \& Gerhard 1994 MNRAS 269, 785

[3] Combes \& Arnaboldi 1996 A\&A 305, 763

[4] Gallagher et al. 2002 ApJ 568, 199

[5] Iodice et al. 2002 AJ 123, 195

[6] Iodice et al. 2003 ApJ 585, 730

[7] Sackett et al. 1994 ApJ 436, 629

[8] Swaters \& Rubin 2003 ApJ 587, L23 